\title{
Prions
}

\section{Walter Bodemer}

German Primate Center, Kellnerweg 4, 37077 Göttingen, Germany

Correspondence to: Walter Bodemer (wbodemer@dpz.eu)

Received: 15 June 2016 - Revised: 24 August 2016 - Accepted: 30 August 2016 - Published: 7 September 2016

\begin{abstract}
Prions gained widespread public and scientific interest in the year 2000. At that time, the human neurological Creutzfeldt-Jakob disease (CJD) was known. However, new CJD cases were diagnosed but they could not be ascribed to one of the classical CJD categories i.e. sporadic (sCJD), hereditary or acquired. Hence, they were classified as variant CJD (vCJD). Later on, experimental evidence suggested that VCJD was caused by prions postulated as unique novel infectious agents and, for example, responsible for bovine spongiform encephalopathy (BSE) also known as mad cow disease. The infection of humans by transmission of BSE prions also defined vCJD as a zoonotic disease. Prions, especially those associated with scrapie in sheep had been known for quite some time and misleadingly discussed as a slow virus. Therefore, this enigmatic pathogen and the transmission of this unusual infectious agent was a matter of a controversial scientific debate. An agent without nucleic acid did not follow the current dogma postulating DNA or RNA as inheritable information encoding molecules. Although numerous experimental results clearly demonstrated the infectious capacity of prions in several animal species, a model close to human was not readily available. Therefore, the use of rhesus monkeys (Macaca mulatta) served as a non-human primate model to elucidate prion infection under controlled experimental conditions. Not the least, transmission of BSE, human vCJD, and SCJD prions could be confirmed in our study. Any prion infection concomitant with progression of disease in humans, especially vCJD, could be analyzed only retrospectively and at late stages of disease. In contrast, the prion-infected rhesus monkeys were accessible before and after infection; the progression from early stage to late clinical stages - and eventually death of the animal - could be traced. Because of the phylogenetic proximity to humans, the rhesus monkey was superior to any rodent or other animal model. For these reasons an experimental approach had been conceived by J. Collinge in London and A. Aguzzi in Zurich and performed in a cooperative study with both research groups in the pathology unit of the German Primate Center (DPZ). The study in the DPZ lasted from 2001 until 2012. Our research in the pathology unit provided a temporal monitoring of how an initial prion infection develops eventually into disease; an approach that would have never been possible in humans since the time point of infection with prions from, for example, BSE is always unknown. Telemetry revealed a shift in sleepwake cycles early on, long before behavioral changes or clinical symptoms appeared. Pathology confirmed nonneuronal tissue as hidden places where prions exist. The rhesus model also allowed first comparative studies of epigenetic modifications on RNA in peripheral blood and brain tissue collected from uninfected and prioninfected animals. To conclude, our studies clearly demonstrated that this model is valid since progression to disease is almost identical to human CJD.
\end{abstract}




\section{Introduction}

\subsection{Past and presence}

Prions are known and now broadly accepted as unusual pathogens since experiments for a long-sought virus were unsuccessful. This novel infectious entity mainly consists of the cellular prion protein (PrP), whose function has not yet been unambiguously identified. As soon as proteinaceous aggregates are formed, either they may confer toxicity to cells or depending on the structure of aggregates they convert into a transmissible pathogen. Human CreutzfeldtJakob disease (CJD) is the most prominent example for a human prion disease and belongs to transmissible spongiform encephalopathies. Several neurologic human diseases like Alzheimer's disease, Parkinson's disease, or amyotrophic lateral sclerosis seem to be caused by cellular proteins switching from a normal physiological function into an abnormal most deleterious function leading to degeneration of cells and tissue. Remarkably, many similarities exist to yeast prions inherited in a non-Mendelian manner. The most prominent example is the non-sense codon suppressing Suppressor35 protein (Sup35 protein). The yeast Sup35 protein is encoded by the SUP 35 gene - a gene which corresponds to the eukaryotic gene coding for one release factor in translation, eRF3. The Sup35 protein can adopt two physiologically relevant states either the "psi - " state or the "PSI +" state responsible for stop of translation or read-through, respectively. During the past years about 100 prion-like proteins in yeast and fungi have been identified and their functional roles can be explained. Finding reasons for an evolutionary role of prionprotein states of these cellular proteins in yeast and fungi is a matter of current research in order to dissect evolutionary versus physiological explanations.

Scrapie as a disease in sheep has been known for centuries. It appeared in ruminants in 1980 and in 2001 after zoonotic transmission of bovine spongiform encephalopathy (BSE) into humans as variant CJD (vCJD). The number of expected human cases based on extrapolation from the first vCJD cases in the UK is uncertain since statistics are insufficient. Fortunately, the vCJD cases remained limited, mounting to 277 cases worldwide in January 2016.

After 20 years of research, experimental evidence has now been accumulated and confirmed many assumptions of the prion "protein-only hypothesis" - a hypothesis that originally evolved from irradiation studies by T. Alper and on a mathematical model by J. S. Griffith in the late 1960s. Further support emerged later from prion transmission experiments by D. C. Gajdusek and C. J. Gibbs. Numerous experimental results were combined by $\mathrm{S}$. Prusiner, and eventually the protein-only hypothesis took shape in its present form. Animal models like mouse strains, hamster, and transgenic mice have been established to study prion infection, progression to disease, and transmission in living organism. In addition, valid cell culture systems for infection have been de- veloped to study initial steps of infection, formation of toxic PrP molecules, and transmissible prion protein aggregates. Recombinantly made PrP could be modified to adopt unique structural variants, such as those with acquired infectious and transmissible capacity. The use of the protein misfolding cyclic amplification (PMCA) became an outstanding molecular technique to discern prion protein structures and their normal functions as well as their aberrant functions in infection and transmission. The PMCA has been derived from studies with prion-like proteins found in yeast. In yeast, nonMendelian inheritance could only be understood assuming prion-like conformational shifts of physiologically relevant proteins like the Sup35 protein.

All experimental data point to zoonosis. BSE was transmitted from cattle via meat products into humans. Since oral uptake of infectious prions was lower and their infectious capacity was reduced in the gastrointestinal tract, the vCJD cases did not reach the expected high number of cases. However, one should be cautious since prions may persist and may be latent for years.

\subsection{Future}

The current number of BSE cases in cattle decreased and remains on a marginal level. However, single cases with different BSE types have recently been identified and most likely will emerge comparatively to human sporadic CJD (sCJD). The cross-species transmission of novel BSE prion types is under investigation in certain mouse strains, transgenic bovinized/humanized mice, and in non-human primates. To date, additional human-BSE-associated vCJD cases have rarely been reported in the past years. However, the scientific progress and molecular details on other neurological human diseases including transmissibility have been tremendously sparked by the prion research itself and the research on yeast/fungi prions. In the past, prions gained public interest as unique transmissible infectious agents (BSE crisis), but their recent scientific value is much higher than originally thought. Prions or prion-like related conformational changes in the respective proteins may be involved in several epigenetic neurological diseases brought about by misfolded proteins and not by epigenetic modifications of DNA or RNA. Understanding the mechanisms leading to conformational changes surprisingly revealed quite different proteins. Some of them are important for regulation of immunity (MAVS: mitochondrial antiviral signaling adaptor), formation of memory (CPEB: cytoplasmic polyadenylation element-binding protein), and the tumor suppressor protein p53. They also follow "conformational transitions". Subsequently, they fundamentally alter their inherent function by this epigenetic modulation. The amazing characteristics of protein epigenetics of other physiologically relevant proteins would not have been detected without mammalian prion protein research and the corresponding yeast prion-like proteins. Details on the biology of prions have comprehensively 
been given in two review articles (Bodemer and Kaup, 2004; Bodemer, 2009).

\section{Methods and results}

\subsection{Animals}

The reason to perform prion research in rhesus monkeys was to monitor infection and the temporal progression of prion infection in the rhesus monkey. In contrast to studies of human CJD cases, we could decide on the infectious dose. We also could control behavior immediately after prion inoculation and during the rather long time until animals died from the prion infection. Hidden places where prions might exist were found. Even epigenetic modifications on RNA could be detected. Taken together, these experimental approaches depended on animals.

Using rhesus monkeys as a model system required thorough ethic reasoning and consultation with authorities before we actually turned to conduct the experiments. The Number of animals was limited just to fulfill statistical conditions. The individual health status was obtained and health care was provided throughout the study. The animals underwent daily inspection to monitor any changes in health and behavior. The experiments were conceived with the aim of reducing pain, suffering, and harm. Groups of animals were preferred in order to keep them in a social environment.

The animals were originally kept in Vienna at Baxter and transferred to the German Primate Center (DPZ) in 2001. J. Collinge, A. Aguzzi, and C. Weissmann were the scientists who recommended this well-controlled prion infection study, and financial support was provided by an EU grant. To ensure statistical significance four groups consisting of four rhesus macaques each were formed: one uninfected control group, one group infected with BSE prions, one with VCJD prions, and one with SCJD prions.

Health of animals, infection, and progression to disease was looked at in our pathology department and in cooperation with W. Schulz-Schaeffer at the UMG (University Medicine, Göttingen). Besides, neurologists from the UMG also observed the animals whenever clinical symptoms seemed to appear. This close observation and comparison with human CJD cases demonstrated how close clinical progression of human disease resembles the experimental infection in the non-human primate.

\subsection{Infection}

Infectious prions from brain tissue of one SCJD and one vCJD case (provided by J. Collinge) as well as BSE prions (from a "German" mad cow case and provided by W. SchulzSchaeffer) were intraperitoneally administered into the rhesus monkeys.

\subsection{Monitoring of behavior and telemetry}

Early behavioral monitoring was carried out by the ethologists I. Machatschke and J. Dittami from Vienna University. Transmitters were used to record changes in the circadian rhythms. Body temperature, sleep-wake cycles, and activity profiles could be obtained over a time span of 2 years. Up to half a year after infection animals did not show any signs of prion infection. However, after 6 months and persisting for another few months some animals had some disturbances in circadian rhythms which disappeared and then never appeared again (I. Machatschke, personal communication, 2006). For a rather long time of about 4-5 years animals seemed to be healthy. But then, almost all animals rapidly progressed to symptoms. Symptoms were highly similar or even identical to those seen in human CJD.

\subsection{Pathology}

Blood and necropsy specimens from the animals served as a valuable source to detect pathologically associated prion protein even in non-neuronal skeletal and cardiac tissue. These "hidden places" of prion pathology and replication were clearly demonstrated and extended our view where prions might spread within an organism. Not only leukocytes and neuronal tissue harbor abnormal prion protein isoforms but also other tissues can propagate prion protein isoforms leading to toxicity, cell degeneration, and eventually transmissible prions (Krasemann et al., 2010, 2013).

\subsection{Therapy}

At late stages we tried to perform a local gene therapy with a recombinant PrP expressing virus shuttle vector. However, it was too late to successfully limit progression to final stages of disease. This study was performed with P. Schucht and D. Ott from A. Aguzzi's group in Zurich.

\subsection{Epigenetics}

Reasoning on epigenetic regulation, we decided to characterize small non-coding RNA in naïve and prion-infected animals. Brain tissue and peripheral cells could be analyzed with respect to quantity and quality of Alu RNA. These RNA species or in other words transcripts were made from repetitive Alu DNA sequences which were defined for decades as junk DNA. Similar to prion disease as an epigenetically directed disease caused by a misfolded protein, we aimed to determine whether Alu transcripts are by themselves epigenetic regulators or are targets for epigenetic modifications of RNA brought about by ADAR (adenosine deaminase acting on RNA) and APOBEC (apolipoprotein B editing complex) editing mechanisms. Both mechanisms act on RNA. ADAR causes deamination of adenosine leading in RNA to an exchange of $A$ into $G$, and APOBEC converts cytidine into uridine leading to an exchange of $\mathrm{C}$ into $\mathrm{U}$ in RNA. Because we 
had necropsy specimens from our monkeys we could characterize side by side Alu RNA with respect to these epigenetic modifications (Kiesel et al., 2012). We also had a chance to analyze BC200 RNA. BC200 RNA plays a role in synaptical function. Of note, we found that BC200 was neither edited in specimens from naïve nor infected rhesus monkeys, whereas Alu RNA underwent extensive editing in both infected and non-infected animals. It may be that BC200 RNA is indispensable in brain cells and should not be altered, whereas Alu RNA is a rather common target for editing. In initial studies we also characterized Alu RNA in specimens from human cases with ambiguous results. Nevertheless, working with rhesus monkeys allowed the introduction of quite a different approach to further differentiate human neuronal diseases like CJD, dementia, or Alzheimer cases referring to small non-coding RNA species.

\subsection{Diagnostics and molecular biology}

Besides our infection study we could extend our previous work on hybridomas producing monoclonal antibodies against animal and human PrP. A most fruitful cooperation with a company developing a BSE test was established. It was highly successful with respect to diagnostics of prion diseases and we received extensive funding to extend our research activities (Yang et al., 2005). In parallel, we turned to molecular determination of the PrP structure in the nanoscale range. With atomic force microscopy (AFM) we could successfully determine the molecular size of a single PrP molecule (Kunze et al., 2008). Recently, our AFM protocol was used to characterize prion protein aggregates which were either toxic or infectious and thus helped to further elucidate the infectious entity of a transmissible prion.

\section{Conclusion}

Most importantly, early signs of an altered circadian rhythm, sleep-wake cycle, and activity and body temperature were recorded in prion-infected animals. This experimental approach would have never been feasible in studies with human CJD cases. After 4-6 years animals developed clinical symptoms highly similar to those typical for CJD. Clinicians confirmed how close the animal model and the human disease matched. Non-neuronal tissue like cardiac muscle and peripheral blood with abnormal, disease-related prion protein were detected in rhesus monkey tissues. Molecular changes in RNA from repetitive Alu and BC200 DNA elements were identified and found to be targets of epigenetic editing mechanisms active in prion disease. To conclude, our results with the rhesus monkey model for prion disease proved to be a valid model and increased our knowledge of pathogenic processes that are distinctive to prion disease.
Acknowledgements. The skillful assistance of our animal care takers, our veterinarians, and my former technicians A. Kues, P. Kiesel, and V. Liebau is greatly appreciated. Many thanks to the EU, BMBF, DFG, and IDEXX for a year-long and substantial funding of our prion research.

Edited by: K. Mätz-Rensing

Reviewed by: two anonymous referees

\section{References}

Bodemer, W.: Prions, Encyclopedia of Microbiology, 3rd Edn., edited by: Schaechter, M., Academic Press, Elsevier, Oxford, 689-706, 2009

Bodemer, W. and Kaup, F. J.: Comments on present-day spread and epidemiology of BSE and prion diseases, Gesundheitswesen, 66, 21-25, 2004

Kiesel, P., Bodemer, W., Gibson, T., Zischler, H., and Kaup, F. J.: Prion infected rhesus monkeys to study differential transcription of Alu DNA elements and editing of Alu transcripts in neuronal cells and blood cells, J. Med. Primatol., 41, 176-182, doi:10.1111/j.1600-0684.2012.00535.x, 2012.

Krasemann, S., Neumann, M., Geissen, M., Bodemer, W., Kaup, F. J., Schulz-Schaeffer, W., Morel, N., Aguzzi, A., and Glatzel, M.: Preclinical deposition of pathological prion protein in muscle of experimentally infected primates, PLoS One, 11, e13906, doi:10.1371/journal.pone.0013906, 2010.

Krasemann, S., Mearini, G., Krämer, E., Wagenführ, K., SchulzSchaeffer, W., Neumann, M., Bodemer, W., Kaup, F. J., Beekes, M., Carrier, L., Aguzzi, A., and Glatzel, M.: BSE-associated prion-amyloid cardiomyopathy in primates, Emerg. Infect. Dis. 19, 985-988, doi:10.3201/eid1906.120906, 2013.

Kunze, S., Lemke, K., Metze, J., Bloukas, G., Kotta, K., Panagiotidis, C. H., Sklaviadis, T., and Bodemer, W.: Atomic force microscopy to characterize the molecular size of prion protein, J. Microsc., 230, 224-232, doi:10.1111/j.13652818.2008.01979.x, 2008.

Yang, W. C., Schmerr, M. J., Jackman, R., Bodemer, W., and Yeung, E. S.: Capillary electrophoresis-based noncompetitive immunoassay for the prion protein using fluorescein-labeled protein A as a fluorescent probe, Anal Chem., 15, 4489-4494, 2005. 